\title{
TFF3 and TIMP3 - the candidate marker genes for differentiation diagnosis of follicular cell-derived thyroid tumors
}

Karolina H. Czarnecka ${ }^{1}$, Michał Kusiński², Maja Kacprzak ${ }^{1}$, Monika Migdalska-Sęk ${ }^{1}$, Ewa Nawrot ${ }^{1}$, Dorota Pastuszak-Lewandoska1 ${ }^{1}$,

Daria Domańska1, Justyna Kiszałkiewicz¹, Krzysztof Kuzdak², Ewa Brzeziańska-Lasota1

${ }^{1}$ Department of Molecular Bases of Medicine, I Chair of Internal Medicine, Medical University of Lodz, Pomorska 251 Str, Lodz, Poland

2 Department of Endocrine, General and Vascular Surgery, Chair of Endocrinology, Medical University of Lodz, Pabianicka 62 Str, Lodz, Poland

\section{Background:}

The study was funded under the grant of the Ministry of Science and Higher Education "luventus Plus" no 0082/IP1/2011/71

Thyroid cancer is a serious epidemiological problem of endocrine diseases. Thyroid carcinoma develops as a result of malignant transformation of nodular goiter (NG), which at an early stage can lead to the development of follicular adenoma (FA). The progression of FA can be associated with the transformation of this benign neoplastic lesion into: papillary thyroid carcinoma (PTC), characterized by slow growth and mild outcome, and thyroid follicular cancer (FTC), the more aggressive form of cancer. The final differentiation of thyroid lesions (FA, PTC, FTC) is usually carried out post-operatively. Therefore, it appears advisable to look for markers enabling the proper preoperative diagnosis.

The search for new differentiating biomarkers in follicular-cell derived thyroid tumors (FCDT), especially for the FNAB with underdetermined cytology, is an important scientific task. Disturbed expression of tumor suppressor genes plays important role in thyroid carcinogenesis.

In this study we focused on epigenetic mechanism influencing on TIMP3 (Tissue Inhibitor Of Metalloproteinases 3) and TFF3 (trefoil factor 3) expression in follicular cell-derived thyroid tumors.

\section{The aim Evaluation gene expression and promoter methylation of TFF3 andTIMP3 as candidate of study: biomarkers in differentiating diagnosis of follicular cell-derived thyroid tumors.}

Material:

* Thyroid neoplasms (N) and matching macroscopically unchanged tissues (Control) from 86 patients with preoperative FNAB diagnosis: PTC/ "follicular neoplasm”.

* Patients aged 16 to 76 years (average 49 years). * 70 women $(81.4 \%)$ and 16 men $(18.6 \%)$.

Table 1. Histopathological classification of patients Histopathological classification

nodular goiter (NG)

follicular adenoma (FA)

carcinoma papillare (PTC)

follicular carcinoma (FTC)

\begin{tabular}{|c|c|lll}
\hline n & $\%$ & \multicolumn{2}{l}{$\begin{array}{l}\text { Memen } \\
\text { Men }\end{array}$} \\
\hline 33 & $38.4 \%$ & 28 & $/ 5$ \\
\hline 9 & $10.5 \%$ & 7 & $/ 2$ \\
\hline 35 & $40.6 \%$ & 29 & $/ 6$ \\
\hline 9 & $10.5 \%$ & 6 & $/ 3$ \\
\hline
\end{tabular}

\section{TIMP3 and TFF3 promoter methylation}

\section{TIMP3 gene}

- presence of both „U” and "M" alleles in all samples ( $n=86,100 \%)$ derived from macroscopically unchanged tissue $(\mathrm{C})$ and thyroid tissue - neoplasms $(\mathrm{N})$

- Highest TIMP3 mean MI was detected in FAs (0.871) and FTCs (0.878)

- TIMP3 MI value was significantly higher in follicular lesions (FTC/FA), than in NGs and PTCs ( $p=0.049$, Kruskal - Wallis test).

\section{TFF3 gene}

- presence of both „U” and „M" alleles in $47.7 \%$ of controls (C) and $54.7 \%$ of neoplasms $(\mathrm{N})$; presence of only „M" allele in $52.3 \%$ of $\mathrm{C}$ and $45.3 \%$ of $\mathrm{N}$

- Highest TFF3 mean MI was detected in FAs (0.916) and NGs (0.913)

- TFF3 MI value revealed the opposite correlation to TIMP3 - low MI in FTC/FA

- In PTC TFF3 MI correlates with RQ level $(p=0.01)$.

\section{Methods:}

* RNA and DNA isolation (Universal RNA Purification Kit - Eurx; QIAamp DNA Mini kit $\left(\right.$ QIAGEN $\left.^{\circledR}\right)$, qualitative and quantitative spectrophotometric analysis of RNA and DNA (BioPhotometer, Eppendorf).

* mRNA expression level (RQ) using Taq Man Low Density Arrays, TaqMan Array Micro Fluidic Card in 7900 HT Fast Real-Time PCR System (Applied Biosystems, USA). Relative gene expression level $R Q=2^{-\Delta \Delta C T}$

* DNA bisulfite conversion (EpiMark® Bisulfite Conversion Kit), followed by promoter methylation level evaluation in methylation specific PCR with methylated and unmethylated primers, Methylation Index (MI) calculation.

- Statistical analysis (Statistica for Windows 10.0)

$\mathrm{IM}=\frac{\mathrm{M}\left[\frac{\mathrm{ng}}{\mathrm{\mu l}}\right]}{\mathrm{U}+\mathrm{M}\left[\frac{\mathrm{ng}}{\mathrm{\mu l}}\right]}$

\section{TFF3 and TIMP3 expression}

TIMP3 gene : highest $R Q$ value among patients with NG (1.823), PTC (1.498), lowest in FTC+FA (1.738), (Kruskal-Wallis test; $p>0.05$ ).

TFF3 gene: highest $R Q$ value among patients with FTC+FA (10.770), PTC (6.949), lowest in NG (4.360), (Kruskal-Wallis test; $\mathrm{p}>0.05$ ).

\section{Correlation of expression and methylation of TIMP3 and TFF3} (Spearman's rank correlation coefficient)

TIMP3 MI correlates with RQ $(p=0.029)$

TIMP3 $\mathrm{MI}$ and $\mathrm{RQ}$ are higher in woman vs. men $(\mathrm{p}=0.016)$.

In PTC TFF3 MI correlates with RQ level $(p=0.01)$.

In women positive correlation between Mls and RQs for TIMP3 and TFF3 were found ( $p=0.0004, p=0.00$ respectively)

- TFF3 and TIMP3 RQ values in groups regarding PTNM groups reversely correlate with $\mathrm{Ml}$ for both genes $(p>0)$

- Significant correlation between methylation levels in TIMP3 and TFF3 $(p=0.00009)$, as well as between expression levels in both genes $(p=0.000000)$.

\section{Conclusions:}

The increased TIMP3 MI values in FA/FTC, TFF3 in PTC and correlation between Mls with RQs suggest that can be regarded as promising biomarkers for distinguishing follicular cell-derived thyroid tumors. Our research indicates that simultaneous analysis of methylation profile and expression level of TIMP3 and TFF3 may be diagnostically useful. Further studies are needed. 